\title{
Andreoni-McGuire Algorithm and the Limits of Warm-Glow Giving
}

\author{
Huseyin Yildirim
}

Duke University

January 26, 2013

\section{ERID Working Paper Number 143}

This paper can be downloaded without charge from the Social Science Research Network Electronic Paper Collection:

http://ssrn.com/abstract=2254111

\section{Economic Research Initiatives at Duke WORKING PAPERS SERIES

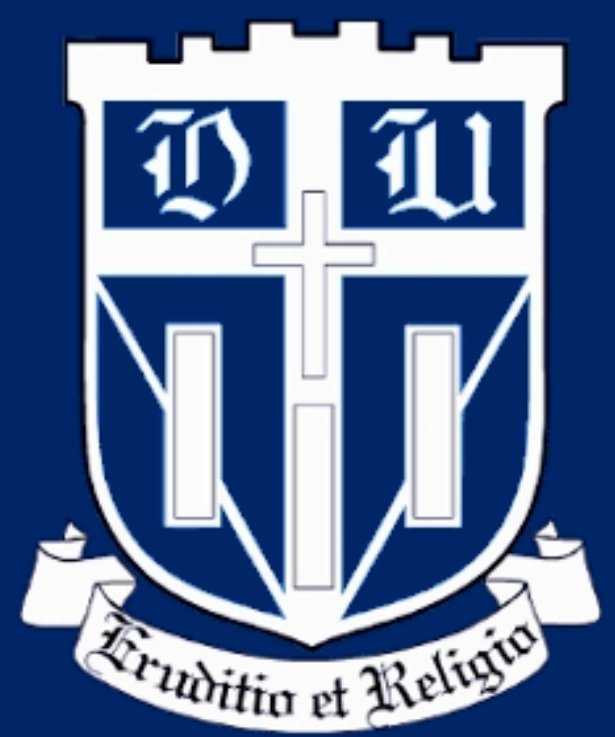




\title{
Andreoni-McGuire Algorithm and the Limits of Warm-Glow Giving*
}

\author{
Huseyin Yildirim \\ Department of Economics \\ Duke University \\ Box 90097 \\ Durham, NC 27708 \\ E-mail: yildirh@econ.duke.edu
}

January 26, 2013

\begin{abstract}
This paper provides a full equilibrium characterization of warm-glow giving à la Andreoni $(1989,1990)$ by extending the Andreoni-McGuire $(1993)$ algorithm. The characterization indexes individuals according to their free-riding or "dropout" levels of the public good. The dropout level is finite for an individual whose donation is always dictated by some altruism. We show that if all individuals have finite dropout levels, then the crowding-out becomes complete as the population size grows. This suggests that in a large economy, the crowding-out is incomplete only when a non-negligible fraction of individuals behaves as though they were pure warm-glow givers. But since these individuals are also the only contributors in a large economy, the incomplete crowding-out must be zero. We discuss implications of these extreme crowding-out predictions for charitable behavior and fund-raising strategies.
\end{abstract}

Keywords: altruism, warm-glow, crowding-out

JEL Classification: H00, H30, H50

\section{Introduction}

Despite its intuitive appeal, a purely altruistic model of giving in which donors care only about the provision of the public good has proved unrealistic. Empirical evidence consistently refutes two chief predictions of this model: complete (dollar-for-dollar) crowdingout of private donations by government grants (Warr, 1983; Bergstrom et al. 1986), and

\footnotetext{
*I thank Jim Andreoni, Ralph Boleslavsky, Paul Dudenhefer, Osman Kocas and Alvaro Name-Correa for comments. All errors are mine.
} 
giving only by the very rich in a large economy (Andreoni, 1988). ${ }^{1}$ To reconcile the evidence, Andreoni $(1989,1990)$ has introduced an additional direct benefit or "warm-glow" from the act of giving itself - calling the extended model warm-glow giving. ${ }^{2}$ Empirical research has strongly corroborated the presence of both altruistic and warm-glow motives for giving. ${ }^{3}$ Despite its prevalent use, a full equilibrium characterization of warm-glow giving is, however, absent in the literature. ${ }^{4}$ This is the gap we fill in this paper. In light of our characterization, we establish a simple necessary and sufficient condition for complete and zero crowding-out in a large warm-glow economy - an issue first considered by Ribar and Wilhelm (2002). As a by-product of our analysis, we also demonstrate that the equilibrium theory of warm-glow giving is as tractable as its polar case of pure altruism.

The key to our equilibrium characterization is to first identify the set of contributors. We achieve this by modifying Andreoni and McGuire's (1993; henceforth A-M) elegant algorithm tailored to pure altruism. The A-M algorithm, and our modification of it, ranks each individual according to her free-riding threshold of the public good, or her "dropout" amount. We show that an individual becomes a contributor (in the whole economy) if and only if the subeconomy consisting of those ranked higher than her fail to supply the public good at her dropout amount. Therefore, one's decision to contribute depends solely on those less likely to free ride than her while her gift size depends on the entire set of contributors. Indeed, the equilibrium public good occurs at the level that neutralizes the marginal contributor's incentive to give.

A noteworthy feature of a person's dropout amount is that it is pinned down by her own preferences and income. We establish that this amount is finite if her donation is always dictated by some altruism. We then prove that if everyone in the economy has a finite dropout amount, in a large economy, the crowding-out must be complete. The reason is that the total equilibrium public good, including any government grants, cannot

\footnotetext{
${ }^{1}$ Most empirical studies find little crowding-out -often 0 to 35\% - (e.g., Kingma, 1989; Okten and Weisbrod 2000; Ribar and Wilhelm, 2002; Manzoor and Straub, 2005; and Gruber and Hungerman, 2007). Moreover, most Americans give. For instance, in 2000, 9 out of 10 U.S. households donated to at least one charitable organization. See Andreoni (2006) and Vesterlund (2006) for an overview of the charitable sector.

${ }^{2}$ See also Cornes and Sandler (1984) and Steinberg (1987).

${ }^{3}$ The incomplete crowding-out observed in empirical studies (see footnote 1 ) is often taken to be the evidence of warm-glow. For experimental evidence of the incomplete crowding-out, see Andreoni, 1993; Bolton and Katok, 1998. Direct experimental tests of warm-glow are, however, also available; e.g., Palfrey and Prisbrey 1997; Andreoni and Miller, 2002; Crumpler and Grossman, 2008; and Korenok et al., 2012.

${ }^{4}$ Andreoni (1989) proves the equilibrium existence and uniqueness by restricting attention to positive contributions. Kotchen (2007) extends the result by accounting for the free-riding possibility. Neither, however, offers a full characterization.
} 
exceed a contributor's dropout amount. And as the population size increases, the public good converges to the highest dropout amount, which is determined independently of government grants. This means that in a large economy, incomplete crowding-out can result only because a non-negligible fraction of the population always gives. But in the limit, such individuals must behave as though they were pure warm-glow givers and in turn the crowding-out must be zero. These extreme crowding-out predictions of the warmglow model are in line with Ribar and Wilhelm's limit analysis; however, our conditions are more general and easier to interpret. Nevertheless, since, on its face, the warm-glow model does not explain partial crowding-out observed in (large-sample) empirical studies, we argue in Section 4 that the model needs to incorporate other important features of the charitable sector such as fundraising and informational asymmetry.

The rest of the paper is organized as follows. We set up the basic model in the next section, and provide an equilibrium characterization in Section 3. In Section 4, we extend the analysis to include government grants and perform a limit economy analysis. In Section 5, we briefly discuss our findings and possible modifications of the model. We conclude in Section 6. Proofs that do not appear in the text are relegated to the appendix.

\section{Warm-Glow Giving}

Consider the model of warm-glow giving studied by Andreoni $(1989,1990)$. An economy consists of $n$ individuals who each allocates her monetary income, $m_{i}>0$, between a private good consumption, $x_{i} \geq 0$, and a gift to the public good or charity, $g_{i} \geq 0$, without observing others' decisions. Both the public and private goods are normal. Units are measured in dollars so that $x_{i}+g_{i}=m_{i}$. Let $G=\sum_{j} g_{j}$ be the supply of the public good, and $u^{i}\left(x_{i}, G, g_{i}\right)$ be a twice continuously differentiable, strictly increasing and strictly quasi-concave utility function for individual $i$. This specification admits as polar cases pure altruism, $u^{i}\left(x_{i}, G\right)$, where individual $i$ cares only about the charity, and pure warm-glow, $u^{i}\left(x_{i}, g_{i}\right)$, where she cares only about the act of giving. In general, individual $i$ enjoys her gift, $g_{i}$, through the public good and also as a private good.

To determine gifts at a Nash equilibrium, we first determine person $i$ 's optimal gift, $\widehat{g}_{i}$, given others' $G_{-i}=\sum_{j \neq i} g_{j}$. Formally, $\widehat{g}_{i}$ solves

$$
\max _{g_{i} \geq 0} u^{i}\left(m_{i}-g_{i}, G_{-i}+g_{i}, g_{i}\right)
$$


The first-order condition for $\widehat{g}_{i}$ is ${ }^{5}$

$$
\frac{d}{d g_{i}} u^{i}\left(m_{i}-g_{i}, G_{-i}+g_{i}, g_{i}\right) \leq 0\left(=0 \text { if } g_{i}>0\right) .
$$

As with Andreoni, an alternative (and often more intuitive) approach to (P) is to substitute for $g_{i}=G-G_{-i}$ :

$$
\max _{G \geq G_{-i}} u^{i}\left(m_{i}+G_{-i}-G, G, G-G_{-i}\right) .
$$

Let $\widehat{G}=\max \left\{f^{i}\left(m_{i}+G_{-i}, G_{-i}\right), G_{-i}\right\}$ be the unique solution to $\left(\mathrm{P}^{\prime}\right)$, where $f^{i}$ is the Nash supply function. Then,

$$
\widehat{g}_{i}=\max \left\{f^{i}\left(m_{i}+G_{-i}, G_{-i}\right)-G_{-i}, 0\right\} .
$$

Let $f_{a}^{i}$ and $f_{w}^{i}$ denote $f^{i}$ s partial derivatives with respect to its first and second terms, signifying person $i$ 's marginal propensity to give due to altruism and due to warm-glow, respectively. Normality of public and private goods imply that $0<f_{a}^{i}<1$ and $f_{w}^{i} \geq 0$. For $f_{w}^{i}=0$, the warm-glow model reduces to pure altruism while for $f_{a}^{i}+f_{w}^{i}=1$, it reduces to pure warm-glow giving. Since we are interested in settings with both motives present, we assume $0<f_{a}^{i}+f_{w}^{i}<1$. From (2), this implies that for $\widehat{g}_{i}>0, \frac{\partial}{\partial G_{-i}} \widehat{g}_{i}=f_{a}^{i}+f_{w}^{i}-1<0$ and $1+\frac{\partial}{\partial G_{-i}} \widehat{g}_{i}=f_{a}^{i}+f_{w}^{i}>0$; that is, others' contribution crowds out one's contribution but only partially. To see what this implies about preferences, note from (1) that

$$
\frac{\partial}{\partial G_{-i}} \widehat{g}_{i}=-\frac{\frac{d}{d g_{i}} u_{G}^{i}(.)}{\frac{d^{2}}{d g_{i}^{2}} u^{i}(.)} .
$$

Since $\frac{d^{2}}{d g_{i}^{2}} u^{i}()<$.0 by the second-order condition of $(\mathrm{P})$, and $\frac{\partial}{\partial G_{-i}} \widehat{g}_{i}<0$, we must have

$$
\frac{d}{d g_{i}} u_{G}^{i}(.)=-u_{x G}^{i}(.)+u_{G G}^{i}(.)+u_{G g_{i}}^{i}(.)<0 .
$$

The condition in (4) holds for a wide range of applications. If $u^{i}$ is concave and separable in private consumption, i.e., $u_{x G}^{i}()=$.0 , then (4) requires $u_{G g_{i}}^{i}()<.-u_{G G}^{i}($.$) , which allows$ substitution and some complementarity between warm-glow and altruistic preferences. For instance, "impact philanthropy" where an individual's warm-glow comes from "making a difference" may mean substitutes, $u_{G g_{i}}^{i}()<0,.{ }^{6}$ because others' contribution reduces

\footnotetext{
${ }^{5}$ The second-order condition is satisfied due to the strict quasi-concavity of $u^{i}$.

${ }^{6}$ See the specification (6) below. Duncan (2004) offers an interesting theory of impact philanthropy, although his definition of "making a difference" requires $u_{G_{-i}}^{i}()<$.0 , which is somewhat different.
} 
the marginal impact of one's own whereas social institutions coaxing donations may mean complements, $u_{G g_{i}}^{i}()>$.0 , because individuals may be concerned about their social images and audience effects. If, on the other hand, $u^{i}$ is concave and separable in warm-glow, i.e., $u_{G g_{i}}^{i}()=$.0 , then (4) requires $u_{G G}^{i}()<.u_{x G}^{i}($.$) , which allows complementarity and some$ substitution between public and private goods. Complementarity may arise, for instance, between watching TV and supporting public TV while substitution may arise between investing in home security and supporting police. ${ }^{7}$

To avoid trivial noncontributing, we assume that each person has a positive standalone demand for the public good, i.e., $f^{i}\left(m_{i}, 0\right)>0$. A necessary and sufficient condition for this is that $\frac{d}{d g_{i}} u^{i}\left(m_{i}, 0,0\right)>0$.

\section{Equilibrium Characterization: Andreoni-McGuire Algorithm Revisited}

By definition, equilibrium gifts lie at the intersection of individuals' reaction functions and can be found by simultaneously solving $n$ equations described by (2). This direct approach, however, yields limited insight into giving behavior. In particular, it does not systematically identify the set of contributors, which is critical to understanding individual incentives to give. Within a purely altruistic model, Andreoni and McGuire (1993) provide an elegant algorithm that does exactly that. We show that their analysis extends to warmglow giving. In the process, we demonstrate that warm-glow giving is as tractable as its special case of pure altruism.

As in Andreoni and McGuire, let $G_{i}^{0}$ denote others' threshold contribution, or the public good level, that induces person $i$ to free ride or "drop out" of the contributor set; that is, $g_{i}=0$ if and only if $G_{-i} \geq G_{i}^{0}$. Eq.(2) then implies,

$$
f^{i}\left(m_{i}+G_{i}^{0}, G_{i}^{0}\right)-G_{i}^{0}=0 .
$$

Since $f_{a}^{i}+f_{w}^{i}-1<0$ and $f^{i}\left(m_{i}, 0\right)>0$, if a solution to (5) exists, it must be unique (and strictly positive). If a solution does not exist, it simply means that person $i$ would never free ride or would, by convention, have $G_{i}^{0}=\infty$. Alternatively, from (4), $G_{i}^{0}$ must satisfy: $\frac{d}{d g_{i}} u^{i}\left(m_{i}, G_{i}^{0}, 0\right)=0$. Since $\frac{d}{d g_{i}} u^{i}\left(m_{i}, 0,0\right)>0, G_{i}^{0}$ exists if and only if $\frac{d}{d g_{i}} u^{i}\left(m_{i}, G, 0\right) \leq 0$

\footnotetext{
${ }^{7}$ Keeping up with Andreoni $(1989,1990)$, here we consider cases where $\frac{\partial}{\partial G_{-i}} \widehat{g}_{i}<0$. It is, however, possible that $\frac{\partial}{\partial G_{-i}} \widehat{g}_{i}>0$, which would imply "crowding-in". Under upward-sloping reaction functions, the equilibrium uniqueness can no longer be guaranteed, but our limit economy results would still hold.
} 
for some $G$. Thus, person $i$ never free rides if and only if $\frac{d}{d g_{i}} u^{i}\left(m_{i}, G, 0\right)>0$ for all $G$; that is, if and only if the warm-glow motive remains active at all levels of the public good. An issue that is especially pertinent to large economies is whether warm-glow becomes the sole charitable motive at very high levels of the public good. The following result shows this is the case.

Proposition 1 Let $\lim _{G_{-i} \rightarrow \infty} f_{a}^{i}+f_{w}^{i}=\theta_{i}$.

(a) If $\theta_{i}<1$, then $G_{i}^{0}<\infty$.

(b) If $G_{i}^{0}=\infty$, then $\lim _{G_{-i} \rightarrow \infty} \frac{\partial}{\partial G_{-i}} \widehat{g}_{i}=0$, and thus $\lim _{G_{-i} \rightarrow \infty} \frac{d}{d g_{i}} u_{G}^{i}\left(m_{i}-\widehat{g}_{i}, \widehat{G}, \widehat{g}_{i}\right)=0$.

(c) Suppose $u^{i}$ is homothetic. Then, $\theta_{i}=f^{i}(1,1)$. For $\theta_{i}=1, \lim _{G_{-i} \rightarrow \infty} \widehat{g}_{i}=f_{a}^{i}(1,1) \times m_{i}$.

Proof. See the appendix.

Recall that pure warm-glow giving refers to $f_{a}^{i}+f_{w}^{i}=1$ or equivalently $\frac{\partial}{\partial G_{-i}} \widehat{g}_{i}=0$ for all $G_{-i}$. Part (a) indicates that even after very generous contribution by others, if a person's last dollar to charity is partially driven by a concern for its output, or altruism, then she will turn a free rider at some threshold donation by others. ${ }^{8}$ Conversely, part (b) says that a person who always gives will grow unresponsive to others' contribution and behave as though she were a pure warm-glow giver. When utility is homothetic, part (c) explicitly shows such a person's asymptotic contribution.

To illustrate Proposition 1, consider these homothetic utilities similar to those used by Ribar and Wilhelm (2002).

$$
\begin{gathered}
u^{i}=(1-\alpha) \ln x_{i}+\alpha \ln \left((1-\omega) G+\omega g_{i}\right) . \\
u^{i}=(1-\alpha-\omega) \ln x_{i}+\alpha \ln G+\omega \ln g_{i} .
\end{gathered}
$$

Under specification (6), $f^{i}=\alpha\left(m_{i}+G_{-i}\right)+\omega(1-\alpha) G_{-i}$, and thus $f_{a}^{i}+f_{w}^{i}=\alpha+\omega(1-$ $\alpha)<1$ and $G_{i}^{0}=\frac{\alpha}{(1-\alpha)(1-\omega)} m_{i}<\infty$. Under specification (7), $f_{a}^{i}+f_{w}^{i} \rightarrow \frac{\omega}{1-\alpha}+\frac{1-\alpha-\omega}{1-\alpha}=1$

\footnotetext{
${ }^{8}$ This is, of course, a counter-factual for person $i$ because she does not observe others' contributions while choosing her own.
} 
and thus $G_{i}^{0}=\infty$ and $\widehat{g}_{i} \rightarrow \frac{\omega}{1-\alpha} m_{i}$ as $G_{-i} \rightarrow \infty .{ }^{9}$ Consistent with part (c), $\widehat{g}_{i}=\frac{\omega}{1-\alpha} m_{i}$ is the optimal gift by a pure warm-glow giver whose utility is $\bar{u}^{i}=(1-\alpha-\omega) \ln x_{i}+\omega \ln g_{i}$.

An important feature of $G_{i}^{0}$ is that it depends on one's own preferences and income not on the equilibrium. Without loss of generality, index individuals in a descending order of their dropout levels:

$$
G_{1}^{0} \geq G_{2}^{0} \geq \ldots \geq G_{n}^{0} .
$$

A pivotal step in generalizing the A-M algorithm to warm-glow giving is to define the inverse function $\phi^{i}\left(G, m_{i}\right)$ from (2):

$$
f^{i}\left(m_{i}+\phi^{i}, \phi^{i}\right)=G
$$

Such an inverse exists because $f_{a}^{i}+f_{w}^{i}>0$. It is readily verified that partial derivatives satisfy $\phi_{G}^{i}=\frac{1}{f_{a}^{i}+f_{w}^{i}}>1$ and $-1<\phi_{m}^{i}=-\frac{f_{a}^{i}}{f_{a}^{i}+f_{w}^{i}}<0$. By definition, $\phi^{i}\left(G, m_{i}\right)$ measures how much person $i$ values others' total contribution, $G_{-i}$, at the public good level $G$. As a result, person $i$ 's optimal gift in (2) can be re-written:

$$
g_{i}=\max \left\{G-\phi^{i}\left(G, m_{i}\right), 0\right\},
$$

and eq. (5) can be re-stated: $G_{i}^{0}-\phi^{i}\left(G_{i}^{0}, m_{i}\right)=0$. Clearly, $i^{\prime}$ s gift is decreasing in $G$ and increasing in $m_{i}$ but both at a lower rate for a warm-glow giver than a pure altruist. The reason is as explained in Andreoni (2006, p. 1221): warm-glow creates a "stickiness" to giving - people are no longer indifferent to the source of the gift. It is worth noting that with pure altruism, $\phi^{i}\left(G, m_{i}\right)=\bar{\phi}^{i}(G)-m_{i}$ where $\bar{\phi}^{i}$ denotes the inverse demand for public good.

Andreoni and McGuire prove and explain five facts to formulate their algorithm. We show that these facts extend to warm-glow giving, and record them here for exposition. Adopting the A-M notation, let $G^{*}$ denote the equilibrium public good and $C$ denote the set of contributors.

Fact $1 i \in C$ if and only if $G^{*}<G_{i}^{0}$.

This fact directly follows from the definition of $G_{i}^{0}$ and (8). It indicates that the equilibrium public good - not just others' contribution - must stay strictly below a contributor's

\footnotetext{
${ }^{9}$ Letting $M_{i}=m_{i}+G_{-i}$, we find

$$
f^{i}\left(M_{i}, G_{-i}\right)=\frac{(\alpha+\omega) M_{i}+(1-\omega) G_{-i}+\sqrt{\left((\alpha+\omega) M_{i}+(1-\omega) G_{-i}\right)^{2}-4 \alpha M_{i} G_{-i}}}{2} .
$$
}

Evidently, $f^{i}(1,1)=1$. 
dropout point. Therefore if $G_{i}^{0}$ is bounded for all $i$, so is the equilibrium public good. This implication will prove instrumental in studying crowding-out. The next two facts are immediate from Fact 1. In particular, Fact 3 establishes that a contributor cannot turn a free-rider when a new person who is more likely to drop out joins the group.

Fact 2 If $i \in C$ and $G_{j}^{0} \geq G_{i}^{0}$, then $j \in C$.

Fact 3 Let $G_{\min }^{0}=\min \left\{G_{i}^{0}\right.$ s.t. $\left.i \in C\right\}$. If $G_{k}^{0} \leq G_{\min }^{0}$ and person $k$ is added to the economy, none of the original members of $C$ will be replaced, although person $k$ may be added to $C$.

Fact 4 makes the obvious point that as new individuals are added to the group, public good provision cannot decrease despite the intensified free riding.

Fact 4 Let $S^{\prime}$ and $S^{\prime \prime}$ be two sets of individuals such that $S^{\prime} \subset S^{\prime \prime}$, with the respective equilibrium levels of public goods, $G^{\prime}$ and $G^{\prime \prime}$. Then, $G^{\prime} \leq G^{\prime \prime}$.

Proof. Suppose, to the contrary, that $G^{\prime}>G^{\prime \prime}$. Then, by Fact $1, C^{\prime} \subseteq C^{\prime \prime}$. Next, for $i \in C^{\prime}, g_{i}^{\prime}=G^{\prime}-\phi^{i}\left(G^{\prime}, m_{i}\right)<G^{\prime \prime}-\phi^{i}\left(G^{\prime \prime}, m_{i}\right)=g_{i}^{\prime \prime}$ because $\phi_{G}^{i}>1$. But, given $C^{\prime} \subseteq C^{\prime \prime}$, this implies $G^{\prime} \leq G^{\prime \prime}$, a contradiction. Thus, $G^{\prime} \leq G^{\prime \prime}$.

The final fact determines the equilibrium public good that can be produced among the contributors, which directly obtains from aggregating (8).

Fact 5 Let $C_{i}=\{1, \ldots, i\}$ be a set of individuals and $G<G_{i}^{0}$. $G$ is an equilibrium in $C_{i}$ if and only if

$$
\Delta_{i}(G, \mathbf{m}) \equiv G-\sum_{j=1}^{i}\left(G-\phi^{j}\left(G, m_{j}\right)\right)=0
$$

Proof. Suppose $G$ is an equilibrium in $C_{i}$. Then $g_{j}=G-\phi^{j}\left(G, m_{j}\right)$ for $j \in C_{i}$. Summing over all $j \in C_{i}$ yields $\Delta_{i}(G, \mathbf{m})=0$. Conversely, suppose $\Delta_{i}(G, \mathbf{m})=0$, and let $g_{j}=$ $G-\phi^{j}\left(G, m_{j}\right)$. Since $G<G_{i}^{0}$, we have $g_{j}>0$ and this gift profile constitutes an equilibrium in $C_{i}$.

Armed with these facts, Proposition 2 generalizes the A-M algorithm for warm-glow giving.

Proposition 2 Let $\Delta_{i}^{0} \equiv \Delta_{i}\left(G_{i}^{0}, \mathbf{m}\right)$. Then,

(a) $\Delta_{1}^{0} \geq \Delta_{2}^{0} \geq \ldots \geq \Delta_{n}^{0}$ (with $\Delta_{i}^{0}=\infty$ if $G_{i}^{0}=\infty$ ). 
(b) $i \in C$ if and only if $\Delta_{i}^{0}>0$.

(c) If $C=\{1, \ldots, k\}$, then the equilibrium public good uniquely solves: $\Delta_{k}\left(G^{*}, \mathbf{m}\right)=0$. In equilibrium, $i \in C$ contributes $g_{i}^{*}=G^{*}-\phi^{i}\left(G^{*}, m_{i}\right)$.

Proof. Note that $\Delta_{i}(G, \mathbf{m})$ is strictly increasing in $G$. Thus

$$
\Delta_{i}^{0} \equiv \Delta_{i}\left(G_{i}^{0}, \mathbf{m}\right) \geq \Delta_{i}\left(G_{i+1}^{0}, \mathbf{m}\right) \geq \Delta_{i+1}\left(G_{i+1}^{0}, \mathbf{m}\right) \equiv \Delta_{i+1}^{0},
$$

where the last inequality follows because $G_{i}^{0}-\phi^{i}\left(G_{i}^{0}, m_{i}\right)=0 \leq G_{i+1}^{0}-\phi^{i}\left(G_{i+1}^{0}, m_{i}\right)$. To complete part (a), note that if $G_{i}^{0}=\infty$, then for $j \leq i, \lim _{G \rightarrow G_{i}^{0}} \frac{G-\phi^{j}\left(G, m_{j}\right)}{G}=\lim _{G \rightarrow G_{i}^{0}} \frac{1-\phi_{G}^{j}\left(G, m_{j}\right)}{1}=$ $\lim _{G \rightarrow G_{i}^{0}}\left(1-\frac{1}{f_{a}^{j}+f_{w}^{j}}\right)=0$, where the last equality obtains since $f_{a}^{j}+f_{w}^{j} \rightarrow 1$ for $G_{j}^{0}=\infty$ by Proposition 1(a). Thus, eq.(9) implies that if $G_{i}^{0}=\infty$, it must be that $\Delta_{i}^{0}=G_{i}^{0}=\infty$, as stated.

To prove part (b), suppose $\Delta_{i}^{0}>0$. Then, since $\Delta_{i}(G, \mathbf{m})$ is strictly increasing in $G$, there is a unique $G_{i}^{*}<G_{i}^{0}$ such that $\Delta_{i}\left(G_{i}^{*}, \mathbf{m}\right)=0$. By Fact $5, G_{i}^{*}$ is an equilibrium among $k=1, \ldots, i$. Since $G_{i}^{*}<G_{i}^{0}$, person $i$ is a contributor in this equilibrium. Fact 3 implies that she must stay a contributor in the whole economy. Conversely, suppose $i \in C$. Then, $G^{*}<G_{i}^{0}$ by Fact 1 , and thus $0 \leq \Delta_{i}\left(G^{*}, \mathbf{m}\right)<\Delta_{i}\left(G_{i}^{0}, \mathbf{m}\right)=\Delta_{i}^{0}$. The last part directly follows from Fact 5 and eq.(8).

As with the A-M algorithm, a systematic approach to characterizing equilibrium is to first pinpoint the set of contributors. Part (a) says that the search for contributors follows the same descending order as their dropout levels of the public good. Person i's incentive to become a contributor is, however, measured by $\Delta_{i}^{0}$ in order to account for the freeriding among the contributors. It is this free-riding that creates a wedge between $G_{i}^{0}$ and $\Delta_{i}^{0}$, which grows as one moves down in the order. Consider individual 1 . If she were alone in the economy, she would contribute because $\Delta_{1}^{0}=G_{1}^{0}>0$. In the presence of individual 1 , individual 2 is less eager to contribute since at the public good level $G_{2}^{0}$, she expects 1 to give $G_{2}^{0}-\phi^{1}\left(G_{2}^{0}, m_{1}\right)>0$, leaving her $G_{2}^{0}-\left[G_{2}^{0}-\phi^{1}\left(G_{2}^{0}, m_{1}\right)\right]$ or equivalently $\Delta_{2}^{0}$ since $G_{2}^{0}-\phi^{2}\left(G_{2}^{0}, m_{2}\right)=0$. Iteratively applied, this logic reveals that after the gifts from $j=$ $1, \ldots, i-1, \Delta_{i}^{0}$ is the residual amount individual $i$ needs to bear in order to reach the public good level $G_{i}^{0}$. Part (b) indicates that $i$ becomes a contributor whenever there is a shortfall, i.e., $\Delta_{i}^{0}>0$. In the special case of pure altruism, recall that $\phi^{i}\left(G, m_{i}\right)=\bar{\phi}^{i}(G)-m_{i}$, and thus $\Delta_{i}^{0}>0$ if and only if $\sum_{j=1}^{i} \bar{\phi}^{j}\left(G_{i}^{0}\right)-(i-1) G_{i}^{0}>\sum_{j=1}^{i} m_{j}$, which is exactly the A-M condition for pure altruism. 
Once the set of contributors is determined from the model primitives, part (c) reveals that the equilibrium supply of the public good $G^{*}$ occurs at the level that drives the marginal contributor's incentive to give down to zero. $G^{*}$ must be strictly less than the marginal contributor's dropout level in order for her to stay as a contributor (see Fact 1). Using $G^{*}$, a contributor $i^{\prime}$ s gift is then $g_{i}^{*}=G^{*}-\phi^{i}\left(G^{*}, m_{i}\right)$ from (8).

Proposition 2 is attractive because it isolates individual incentives to give. As alluded to in the Introduction, the characterization shows that one's decision to become a contributor depends solely on those less likely to free ride than her while her actual contribution depends on the entire set of contributors. The characterization can also be used to easily reproduce previous results such as Proposition 1 in Andreoni (1990) on income redistribution and nonneutrality of public good provision: simply assume everyone is a contributor and totally differentiate $\Delta_{n}\left(G^{*}, \mathbf{m}\right)=0$, and then note that $-\phi_{m}^{i}=\frac{f_{a}^{i}}{f_{a}^{i}+f_{w}^{i}}$ is Andreoni's "altruism coefficient". Using Proposition 2, one can, however, extend Andreoni's finding even when free-riding is possible. Our equilibrium characterization of warm-glow giving is also amenable to extensions. In the next section, we include government grants and investigate the issue of crowding-out.

\section{Crowding-out in Limit Economies}

To what degree do government grants crowd out private donation? This is a fundamental question in public economics because it shapes policy and helps understand charitable motives. The evidence is, however, mixed. While empirical studies find small crowdingout -often 0 to $35 \%$, - experimental data shows otherwise - up to $70 \%$ - (see footnotes 1 and 2). This discrepancy has mostly been attributed to donor preferences for giving. In particular, empirical studies point to a stronger warm-glow motive. In an interesting paper, Ribar and Wilhelm (2002) have offered a positive reconciliation of the conflicting evidence based on the sample size differences between field and lab data. By performing a limit analysis of warm-glow giving, they show that under reasonable conditions, the crowding-out can be either complete or zero. Though enlightening, the generality of Ribar and Wilhelm's conditions is difficult to ascertain as they depend on equilibrium gifts. ${ }^{10}$

\footnotetext{
${ }^{10}$ With identical individuals, Ribar and Wilhelm determine the rate at which the equilibrium value of $\left(f_{a}+\right.$ $\left.f_{w}-1\right)$ converges. If the rate is faster than $n$, then the crowding-out is zero; if it is slower than $n$, the crowdingout is complete. They also provide a sufficient condition for the zero crowding-out, which is slightly more restrictive than that: $\frac{d}{d g_{i}} u^{i}\left(m_{i}, G, 0\right)>0$ for all $G$.
} 
By augmenting Proposition 2, we re-address the issue of the crowding-out and derive a simple criterion for the limit crowding-out: if $G_{i}^{0}$ is finite for all $i$, then crowding-out must be complete in the limit; if, on the other hand, $G_{i}^{0}=\infty$ for a nonnegligible percentage of the population, then crowding-out is zero.

Suppose the charity receives a direct government grant $R \geq 0$, which raises the public good supply to $G+R$. Person $i$ 's maximization then becomes

$$
\max _{g_{i} \geq 0} u^{i}\left(m_{i}-g_{i}, G+R, g_{i}\right)
$$

Letting $\bar{G}=G+R, \bar{G}_{-i}=G_{-i}+R$, and $g_{i}=\bar{G}-\bar{G}_{-i}$,

$$
\max _{\bar{G} \geq \bar{G}_{-i}} u^{i}\left(m_{i}+\bar{G}_{-i}-\bar{G}, \bar{G}, \bar{G}-\bar{G}_{-i}\right)
$$

From here, $i$ 's optimal gift is found to be $g_{i}=\max \left\{f^{i}\left(m_{i}+\bar{G}_{-i}, \bar{G}_{-i}\right)-\bar{G}_{-i}, 0\right\}$, or equivalently

$$
g_{i}=\max \left\{\bar{G}-\phi^{i}\left(\bar{G}, m_{i}\right), 0\right\} .
$$

Eq.(10) closely tracks (8), which enables us to reproduce the five facts above by replacing $G^{*}$ with $\bar{G}^{*}$, and incorporate the grant into Proposition 2.

Proposition 3 Let $R \geq 0$ be the government grant. Then,

(a) $i \in C$ if and only if $\Delta_{i}^{0}-R>0$.

(b) If $C=\{1, \ldots, k\}$, then the equilibrium public good uniquely solves: $\Delta_{k}\left(\bar{G}^{*}, \mathbf{m}\right)-R=0$. In equilibrium, $i \in C$ contributes $g_{i}^{*}=\bar{G}^{*}-\phi^{i}\left(\bar{G}^{*}, m_{i}\right)$.

(c) If $G_{1}^{0}>R$, then $G^{*}$ is strictly decreasing in $R$, but $\bar{G}^{*}$ is strictly increasing in $R$.

Proof. See the appendix.

To understand Proposition 3, simply treat the government as another contributor whose gift $R$ lowers person $i$ 's shortfall for the public good level $G_{i}^{0}$ to $\Delta_{i}^{0}-R$. Part (a) says that with the grant, person $i$ is less likely to contribute. That is, the larger the grant, the smaller the set of contributors will be. As in the case without the grant, the equilibrium public good drives the marginal giver's incentive to zero. More importantly, part (c) records that 
in a finite economy, private giving is partially crowded out: the grant displaces some private giving but the total provision increases. ${ }^{11}$ The obvious question, however, remains: what is the degree of the crowding-out in a large economy? The following result answers this question. In its exposition, we call donor $i$ type $t$ if her dropout amount is $G^{t, 0}$. Recall from (5) that two donors with different preferences and different incomes can possess the same dropout amount.

Proposition 4 Without loss of generality, let $G^{1,0}>G^{2,0}>\ldots>G^{T, 0}$ represent donor types, and $\lambda_{t} \neq 0$ be the fraction of type $t$. Also let $G^{1,0}>R$. Then, as $n \rightarrow \infty$,

(a) only type 1 donors contribute;

(b) (complete crowding-out) if $G^{1,0}<\infty$, then $G^{*}(n, R)+R \rightarrow G^{1,0}$ and $g_{i}^{1 *}(n, R) \rightarrow 0$;

(c) (zero crowding-out) if $G^{1,0}=\infty$, each type 1 donor's gift becomes independent of $R$. If, in addition, $u^{i, 1}$ is homothetic, then $g_{i}^{1 *}(n, R) \rightarrow f_{a}^{i, 1}(1,1) \times m_{i}$, which would be the contribution of a pure warm-glow giver.

Proof. Since $\Delta_{i}^{0}=G^{1,0}>R$, all type 1 individuals are contributors for any $n$. If $G^{2,0} \leq R$, clearly no other type contributes. Suppose $G^{2,0}>R$ and, to the contrary, some type $t \neq 1$ remains a contributor as $n \rightarrow \infty$. Then, $G^{*}(\infty, R)+R \leq G^{2,0}$ by Fact 1 . But since $G^{2,0}<G^{1,0}$, type 1 individuals would give a strictly positive amount in the limit, implying that $G^{*}(\infty, R)+R=\infty>G^{2,0}$, a contradiction. Thus, no type other than 1 should contribute in a limit economy. Next, if $G^{1,0}<\infty$, a similar limit argument shows that $G^{*}(n, R)+R \rightarrow G^{1,0}$ as $n \rightarrow \infty$. Since $G^{1,0}$ is independent of $R$, this means complete crowding-out. Moreover, from Proposition $3 b, g_{i}^{1 *}(n, R) \rightarrow G^{1,0}-\phi^{i}\left(G^{1,0}, m_{i}\right)=0$, since $G^{1,0}<\infty$.

Finally, suppose $G^{1,0}=\infty$. Then, as $n \rightarrow \infty, G^{*}(n, R) \rightarrow \infty$; otherwise, $G^{*}(\infty, R)=$ $G_{l}<\infty$ would imply $g_{i}^{1 *}(\infty, R)=0$ for some person $i$ of type 1 , which would imply $\left(G_{l}+R\right)-\phi^{i}\left(G_{l}+R, m_{i}\right)=0$ by (10). But then, there must be some $G>G_{l}$ such that $(G+R)-\phi^{i}\left(G+R, m_{i}\right)<0$, contradicting $G^{1,0}=\infty$. Since $G^{*}(n, R) \rightarrow \infty$, so is $G^{*}(n, R)+$ $R \rightarrow \infty$. From (10), this means that $g_{i}^{1 *}(\infty, R)$ is independent of $R$. If, in addition, $u_{i}^{1}$ is

\footnotetext{
${ }^{11}$ Note that we ignore the financing issue of the government grant here. As such, Proposition 3 slightly underestimates the crowding-out. If person $i$ is taxed $\tau_{i}$ to finance $R$, it can be shown that our analysis will still hold except that $G_{i}^{0}$ will use the reduced income $m_{i}-\tau_{i}$.
} 
homothetic, Proposition 1c reveals that $g_{i}^{1 *}(n, R) \rightarrow f_{a}^{i, 1}(1,1) \times m_{i}$, which would be the amount by a pure warm-glow giver.

Proposition 4 makes two points. First, as the economy grows large, only the "most willing" individuals contribute. The reason is as explained in Proposition 1: donors with a finite dropout must feel some altruism and ultimately free ride at a sufficiently high level of the public good that is reached in a large economy. ${ }^{12}$ As a result, the degree of the crowding-out in a large economy is determined by the most willing type's motives for giving. If these donors' altruism is also persistent so that their dropout amount is finite, then the total provision of the public good including the grant asymptotically approaches this amount, $G^{1,0}$. Since $G^{1,0}$ is independent of $R$, this implies that partial crowding-out identified for a finite economy becomes complete in a limit economy. The intuition is that due to the residual altruism, a type 1 donor's contribution is crowded out some by each additional donor and driven to zero in the limit. This means that for the crowding-out to be incomplete, no altruism should be left at high levels of the public good. Indeed, from Proposition 1b, we know that individuals who continue to give at high public good levels must behave as though they were pure warm-glow givers. This in turn implies that in a large economy, the incomplete crowding-out can only be no crowding-out at all. With homothetic preferences, the last part of Proposition 4 shows the exact asymptotic contribution of each individual. As is clear, limit contributions can vary across donors, depending on their warm-glow preferences and incomes.

To illustrate Proposition 4, suppose donors are identical so that $G_{i}^{0}=G^{0}$ for all $i$ (there is only one type). Consider first the utility in (6). Routine computation shows

$$
G^{*}(n, R)=\theta_{n}\left(G^{0}-R\right) \text { and } G^{*}(n, R)+R=\theta_{n} G^{0}+\left(1-\theta_{n}\right) R,
$$

where $G^{0}=\frac{\alpha}{(1-\alpha)(1-\omega)} m$ and $\theta_{n}=\frac{1}{1+\frac{\omega+\alpha(1-\omega)}{n(1-\omega)(1-\alpha)}} \in(0,1)$. Clearly, $\frac{\partial}{\partial R} G^{*}(n, R)=-\theta_{n}$ and $\frac{\partial}{\partial R}\left(G^{*}(n, R)+R\right)=1-\theta_{n}$ (partial crowding-out for a fixed $\left.n\right)$, but $G^{*}(n, R) \rightarrow G^{0}-R$ as $n \rightarrow \infty$ (complete crowding-out in the limit).

Consider next the utility in (7). Directly calculating the equilibrium gifts, we find:

$$
g^{*}=\frac{(n \omega+\alpha) m-(1-\alpha) R+\sqrt{((n \omega+\alpha) m-(1-\alpha) R)^{2}+4 \omega(\alpha+(1-\alpha) n) R m}}{2(\alpha+(1-\alpha) n)} .
$$

\footnotetext{
${ }^{12}$ This observation also generalizes Andreoni's (1988) limit result for pure altruism (Theorem 1.1) to warmglow giving. Specifically, with identical tastes, Proposition 4 implies that only the very rich contribute, though with warm-glow, the average donation need not converge to zero.
} 
As $n \rightarrow \infty, g^{*} \rightarrow \frac{\omega}{1-\alpha} m$, which, as predicted by Proposition 1, would be the gift by a pure warm-glow giver whose utility is $\bar{u}^{i}=(1-\alpha-\omega) \ln x_{i}+\omega \ln g_{i}$. Thus, there is zero crowding-out in this case.

Within these examples, we can also illustrate that the limit results are likely to take effect in relatively small economies. Note that the rate of crowding-out is $r_{n} \equiv\left|\frac{\partial}{\partial R} G^{*}(n, R)\right| \in$ $(0,1)$. For utility (6), let $m=100, \alpha=.03, \omega=.5$ and $R=5$. Then, $r_{n}=\theta_{n}$, and $r_{n}=.90$, .98 , and .99 for $n=10,50$, and 100, respectively; that is, crowding-out is almost complete for $n=100$. For utility (7), let $m=100, \alpha=.03, \omega=.01$ and $R=5$. Then, $r_{n}=.13, .05$ and .03 for $n=10,50$, and 100, respectively; that is, crowding-out is almost zero for $n=100$.

\section{Discussion}

Taken at face value, Proposition 4 implies that if each donor has in mind a "benchmark" level of the public good to be provided, then crowding out should be complete in large sample data. Such a benchmark for the donor exists if altruism or concern for the charity's output always plays a role in her giving decision (Proposition 1). Thus, any hope of explaining the incomplete crowding-out observed in empirical data would require that giving be driven purely by warm-glow and in turn, no crowding-out should occur. ${ }^{13}$ Such an all-or-nothing crowding-out hypothesis does not, however, reconcile theory and evidence on charitable giving.

An alternative interpretation of Proposition 4 is that a theory based only on the donor side is inadequate and the fundraiser side should be an integral part of it. Such added realism opens up a new channel of crowding-out due to the fundraiser behavior. Andreoni and Payne (2011a,b) confirm this view: while estimating 73\% to 100\% total crowding-out, they attribute almost all to reduced fundraising. Thus, a significant crowding-out need not be inconsistent with a strong warm-glow motive for donors. ${ }^{14}$

Another interpretation of Proposition 4 is that the theory should account for informational asymmetries. For one, donors may be uncertain about the size of government aid and thus unable to tailor their donations to the grant. Such uncertainty is, however, unlikely to explain the incomplete crowding-out if the charity itself is perfectly informed of the grant and can costlessly disclose it, perhaps through its website. ${ }^{15}$ The reason is that

\footnotetext{
${ }^{13}$ This is the route taken by Ribar and Wilhelm (2002).

${ }^{14}$ Complementing these studies is the theoretical investigation of Name-Correa and Yildirim (2012).

${ }^{15}$ An important assumption here is that the charity cannot forge the grant information, which seems reason-
} 
the charity that receives little grant will have an incentive to disclose this information to boost private giving. This means that any time the grant information is not disclosed, donors are likely to believe that there is generous government support. This belief then leads to full disclosure of the grant at all levels, much like in the literature on signaling a verifiable quality, e.g., Grossman (1981). The full disclosure may, however, not be possible if there is a nontrivial disclosure cost and/or it is too costly for donors to process the grant information, in which case the uncertainty in grant size may play a role in the degree of crowding-out.

Perhaps an equally important source of informational asymmetry is that individuals are unsure about the quality of the charity, and a government grant can provide a valuable signal - a point first made by Vesterlund (2003). ${ }^{16}$ This signaling probably works best in settings where donors evaluate the charity's qualifications similarly; that is, they at least agree on what constitutes a "good" charity. It is, however, possible that people may have diverse views. Krasteva and Yildirim (2012) consider such a setting where donors acquire costly information about their private valuations of a charity. These authors show that a government grant in this case can cause "classical" crowding-out as well as informational crowding-out, which leaves donors less informed - a donor who is likely to contribute a smaller amount is also less likely to be curious about the charity's project.

\section{Conclusion}

In this paper, we have offered a full equilibrium characterization of warm-glow giving. The important features of this characterization are that it systematically identifies individual incentives to give and it is conducive to comparative statics. It is also open to future extensions, as partly demonstrated by including government grants here and by including fundraising in Name-Correa and Yildirim (2012). Our analysis has heavily utilized individuals' dropout amounts, which reflect their incentives to contribute. Future empirical research may focus on eliciting these dropout amounts when it is difficult to elicit preferences and the access to income data is limited.

\footnotetext{
able, as most grants are awarded as written contracts.

${ }^{16}$ Indeed, several studies have found evidence of such government signaling, e.g., Khanna and Sandler (2000) for the UK charities; Payne (2001) for academic research institutions; and Hautel (2012) for younger charities in the U.S.
} 


\section{$7 \quad$ Appendix}

Proof of Proposition 1. To ease notation, let $z=G_{-i}$ in this proof. Define $F_{i}(z)=f^{i}\left(m_{i}+\right.$ $z, z)-z$. Since $f_{a}^{i}+f_{w}^{i}<1$, clearly $F_{i}^{\prime}(z)<0$. Moreover, since $F(0)=f^{i}\left(m_{i}, 0\right)>0$, there is a unique solution to $F_{i}(z)=0$ if and only if $F_{i}(z) \leq 0$ for some $z<\infty$. We consider two cases. If $\lim _{z \rightarrow \infty} f^{i}\left(m_{i}+z, z\right)<\infty$, there obviously exists such a $z<\infty$. Suppose $\lim _{z \rightarrow \infty} f^{i}\left(m_{i}+z, z\right)=\infty$. Employing l'Hospital's rule, $\lim _{z \rightarrow \infty} \frac{f^{i}\left(m_{i}+z, z\right)}{z}=\lim _{z \rightarrow \infty} \frac{f_{a}^{i}+f_{w}^{i}}{1}=$ $\theta_{i}<1$. Then, because $F_{i}(z)=z\left[\frac{f^{i}\left(m_{i}+z, z\right)}{z}-1\right]$, there must again exist some $z<\infty$ for which $F_{i}(z)<0$.

To prove part (b), suppose $G_{i}^{0}=\infty$. Then, $\theta_{i}=1$ by part (a), and $\lim _{G_{-i} \rightarrow \infty} \frac{\partial}{\partial G_{-i}} \widehat{g}_{i}=0$ by (2), which in turn implies $\lim _{G_{-i} \rightarrow \infty} \frac{d}{d g_{i}} u_{G}^{i}\left(m_{i}-\widehat{g}_{i}, \widehat{G}, \widehat{g}_{i}\right)=0$ by (3).

To prove part (c), suppose $u^{i}$ is homothetic. It can be verified that $f^{i}\left(m_{i}+z, z\right)$ is then homogenous of degree 1 and in turn, $f_{a}^{i}$ and $f_{w}^{i}$ are each homogenous of degree 0 in $\left(m_{i}, z\right)$. The latter reveals that $f_{a}^{i}\left(m_{i}+z, z\right)=f_{a}^{i}\left(\frac{m_{i}}{z}+1,1\right)$ and $f_{w}^{i}\left(m_{i}+z, z\right)=f_{w}^{i}\left(\frac{m_{i}}{z}+1,1\right)$. Therefore, $\theta_{i}=f_{a}^{i}(1,1)+f_{w}^{i}(1,1)$. Next, homogeneity of $f^{i}$ implies that $f^{i}\left(m_{i}+z, z\right)=$ $z f^{i}\left(\frac{m_{i}}{z}+1,1\right)$. Moreover, by Euler's equation, we have $f^{i}=f_{a}^{i} \times\left(m_{i}+z\right)+f_{w}^{i} \times z$ and thus

$$
f^{i}\left(\frac{m_{i}}{z}+1,1\right)=f_{a}^{i}\left(\frac{m_{i}}{z}+1,1\right) \times\left(\frac{m_{i}}{z}+1\right)+f_{w}^{i}\left(\frac{m_{i}}{z}+1,1\right) .
$$

As $z \rightarrow \infty$, clearly $f^{i}(1,1)=f_{a}^{i}(1,1)+f_{w}^{i}(1,1)$ and thus $\theta_{i}=f^{i}(1,1)$, as stated. To complete the proof, note that $F(z)=z\left[f^{i}\left(\frac{m_{i}}{z}+1,1\right)-1\right]$. Suppose $G_{i}^{0}<\infty$ but, to the contrary,

$\theta_{i}=f^{i}(1,1)=1$. Using $1^{\prime} H o s p i t a l^{\prime}$ s rule, $\lim _{z \rightarrow \infty} F(z)=\lim _{z \rightarrow \infty} \frac{f^{i}\left(\frac{m_{i}}{z}+1,1\right)-1}{1 / z}=f_{a}^{i}(1,1) m_{i}$, which is strictly positive and contradicts $G_{i}^{0}<\infty$. Hence, $\theta_{i}<1$. Next suppose $\theta_{i}=1$. Then, $G_{i}^{0}=\infty$, which means person $i$ contributes for any $z$, and by the same limit argument, as $z \rightarrow \infty, \widehat{g}_{i} \rightarrow f_{a}^{i}(1,1) m_{i}$, as recorded. To see that this would be the contribution by a pure warm-glow giver, recall that such a person would satisfy: $f_{a}^{i}+f_{w}^{i}=1$ for all $z \geq 0$. Homogeneity would then imply $F(z)=f_{a}^{i}\left(\frac{m_{i}}{z}+1,1\right) \times m_{i}$, and $\lim _{z \rightarrow \infty} F(z)=f_{a}^{i}(1,1) m_{i}$.

Proof of Proposition 3. Using (10), Facts 1-4 easily extend by replacing $G$ with $\bar{G}$. To see Fact 5, suppose $\bar{G}$ is an equilibrium in $C_{i}$. Then, $g_{j}=\bar{G}-\phi^{j}\left(\bar{G}, m_{j}\right)$ for $j \in C_{i}$. Summing over all $j \in C_{i}$ yields $\Delta_{i}(\bar{G}, \mathbf{m})-R=0$. Conversely, suppose $\Delta_{i}(\bar{G}, \mathbf{m})-R=0$, and let $g_{j}=\bar{G}-\phi^{j}\left(\bar{G}, m_{j}\right)$. Since $\bar{G}<G_{i}^{0}$, we have $g_{j}>0$ and this profile constitutes an equilibrium. Parts (a) and (b) mimic parts (b) and (c) of Proposition 2, respectively.

To prove part (c), suppose $G_{1}^{0}>R^{\prime}>R^{\prime \prime}$. From part (a), we have $\varnothing \neq C^{\prime} \subseteq C^{\prime \prime}$ 
or $1 \leq k^{\prime} \leq k^{\prime \prime}$. Suppose, to the contrary, that $\bar{G}^{* \prime \prime} \geq \bar{G}^{* \prime}$. Then, by Fact $1, k^{\prime \prime} \leq k^{\prime}$ and thus $k^{\prime \prime}=k^{\prime}=k$. This means that $\Delta_{k}\left(\bar{G}^{* \prime}, \mathbf{m}\right)=R^{\prime}$ and $\Delta_{k}\left(\bar{G}^{* \prime \prime}, \mathbf{m}\right)=R^{\prime \prime}$ by part (b), which, since $\Delta_{k}(G, \mathbf{m})$ is strictly increasing in $G$, imply $\bar{G}^{* \prime \prime}<\bar{G}^{* \prime}-$ a contradiction. Hence, $\bar{G}^{* \prime \prime}<\bar{G}^{* \prime}$. From part (b), this implies $g_{i}^{* \prime}<g_{i}^{* \prime \prime}$ for $i=1, \ldots, k^{\prime}$, and since $k^{\prime} \leq k^{\prime \prime}$, we have $G^{* \prime}<G^{* \prime \prime}$. 


\section{References}

[1] Andreoni, J. (1988). "Privately provided public goods in a large economy: the limits of altruism," Journal of Public Economics, 35, 57-73.

[2] Andreoni, J. (1989). "Giving with impure altruism: applications to charity and Ricardian equivalence," Journal of Political Economy, 97, 1447-58.

[3] Andreoni, J. (1990). "Impure altruism and donations to public goods: a theory of warm-glow giving," Economic Journal 100, 464-477.

[4] Andreoni, J. (1993). "An experimental test of the public-goods crowding-out hypothesis," American Economic Review 83, 1317-1327.

[5] Andreoni, J. (2006). Philanthropy, in S-C. Kolm and J. Mercier Ythier, eds., Handbook of Giving, Reciprocity and Altruism, Amsterdam: North Holland: 1201-69.

[6] Andreoni, J. and M. McGuire (1993). “Identifying the Free riders. A Simple Algorithm for Determining who will Contribute to a Public Good," Journal of Public Economics, $51,447-54$.

[7] Andreoni, J. and J.H. Miller (2002). "Giving According to GARP: An Experimental Test of the Consistency of Preferences for Altruism," Econometrica 70(2), 737-53.

[8] Andreoni, J. and A. Payne (2011a). "Is Crowding Out Due Entirely to Fundraising? Evidence from a Panel of Charities," Journal of Public Economics, 95, 334-43.

[9] Andreoni, J. and A. Payne (2011b). "Crowding-Out Charitable Contributions in Canada: New Knowledge from the North," Working Paper.

[10] Bergstrom, T. , L. Blume, and H. Varian (1986). “On the Private Provision of Public Goods," Journal of Public Economics, 29, 25-49.

[11] Bolton, G.E., and E. Katok (1998). “An experimental test of the crowding out hypothesis: the nature of beneficent behavior," Journal of Economic Behavior and Organization 37, 315-331.

[12] Cornes, R., and T. Sandler (1984). "Easy riders, joint production, and public goods," Economic Journal, 94, 580-98. 
[13] Crumpler, H., and P. Grossman (2008). "An experimental test of warm glow giving," Journal of Public Economics, 92, 1011-21.

[14] Duncan, B. (2004). A theory of impact philanthropy. Journal of Public Economics, 88(9), 2159-2180.

[15] Grossman, S. (1981). "The Informational Role of Warranties and Private Disclosure about Product Quality," Journal of Law and Economics, 24(3), 461-83.

[16] Gruber, J., and D. Hungerman (2007). "Faith-based charity and crowd-out during the Great Depression," Journal of Public Economics 91, 1043-69.

[17] Hautel, G. "Crowding Out and Crowding In of Private Donations and Government Grants." Public Finance Review, Forthcoming.

[18] Khanna, J., and T. Sandler (2000). "Partners in Giving: The Crowding-In Effects of UK Government Grants." European Economic Review, 44(8), 1543-56.

[19] Kingma, B. (1989). "An accurate measurement of the crowd-out effect, income effect, and price effect for charitable contributions," Journal of Political Economy, 97, 1197-207.

[20] Korenok, O., E. Millner, and L. Razzolini. "Impure Altruism in Dictators' Giving," Journal of Public Economics, Forthcoming.

[21] Kotchen, M. J. (2007). “Equilibrium existence and uniqueness in impure public good models," Economics Letters, 97(2), 91-6.

[22] Krasteva, S., and H. Yildirim. "(Un)Informed Charitable Giving," Working Paper, 2012.

[23] Manzoor, S., J. Straub (2005). “The robustness of Kingma's crowd-out estimate: evidence from new data on contributions to public radio," Public Choice 123, 463-76.

[24] Name-Correa, A., and H. Yildirim. "A Theory of Charitable Fundraising with Costly Solicitations," American Economic Review, Forthcoming.

[25] Okten, C., B. Weisbrod (2000). "Determinants of donations in private nonprofit markets," Journal of Public Economics 75, 255-272. 
[26] Palfrey, T. R., and J. E. Prisbrey (1997). “Anomalous behavior in public goods experiments: How much and why?" American Economic Review 87(5), 829-46.

[27] Payne, A. (2001). "Measuring the effect of federal research funding on private donations at research universities: is federal research funding more than a substitute for private donations?". International Tax and Public Finance 8, 731-751.

[28] Ribar, D. and M. Wilhelm (2002). "Altruistic and joy-of-giving motivations in charitable behavior," Journal of Political Economy, 110, 425-57.

[29] Roberts, R. (1984). "A positive model of private charity and public transfers," Journal of Political Economy, 92, 136-48.

[30] Steinberg, R. (1987). "Voluntary donations and public expenditures in a federalist system," American Economic Review, 77, 24-36.

[31] Vesterlund, L. (2003). “The Informational Value of Sequential Fundraising," Journal of Public Economics, 2003, 87, 627- 57.

[32] Vesterlund, L. (2006). Why do people give? , in (W. Powell and R.S. Steinberg, eds.), The Nonprofit Sector: A Research Handbook, 2nd Edition, 568-87, New Haven, CT: Yale University.

[33] Warr, P. (1983). "The private provision of a public good is independent of the distribution of income," Economics Letters, 13, 207-11. 